\title{
NEW "CLICKABLE" POLYMERIC COATING FOR GLYCAN MICROARRAYS
}

Caterina Zilio1, Anna Bernardi2, Alessandro Palmioli2, Matteo Salina3, Giovanni Tagliabue4, Marco Buscaglia4, , Roberto Consonni5, Marcella Chiari1*

1 Consiglio Nazionale delle Ricerche, Istituto di Chimica del Riconoscimento Molecolare, 20131 Milano Italy

2 Università degli Studi di Milano, Dipartimento di Chimica, 20133 Milano Italy

3 Proxentia S.r.l., 20135 Milano, Italy

4 Università degli Studi di Milano, Dipartimento di Biotecnologie Mediche e Medicina Traslazionale, 20090 Segrate, Italy

5 Consiglio Nazionale delle Ricerche, Istituto per lo Studio delle Macromolecole, 20133 Milano, Italy

*Corresponding author

marcella.chiari@icrm.cnr.it

Tel. number: +390228500035

Fax number: +390228901239

Keywords: click-chemistry, surface coating, glycan, microarrays

\begin{abstract}
The interaction of carbohydrates with a variety of biological targets, including antibodies, proteins, viruses and cells are of utmost importance in many aspects of biology. Glycan microarrays are increasingly used to determine the binding specificity of glycan-binding proteins. In this study, a novel slide is reported for the fabrication of glycan arrays that
\end{abstract}


combines the higher sensitivity of a layered $\mathrm{Si}^{-\mathrm{SiO}_{2}}$ with a novel approach to form a polymeric coating easily modifiable by subsequent click reaction. The alkyne-containing copolymer, adsorbed from an aqueous solution, produces a coating by a single step procedure and serves as a soft, tridimensional support for the oriented immobilization of carbohydrates via azide/alkyne $\mathrm{Cu}$ (I) catalyzed "click" reaction. The equilibrium and kinetics parameters of the interaction of Concanavalin A with eight synthetic glycans were determined using fluorescence microarray and Reflective Phantom Interface (RPI), a recently proposed optical label-free detection approach. The enhancement of fluorescence provided by the $\mathrm{Si}^{-\mathrm{SiO}_{2}}$ slides enabled to extend the limit of detection at lower surface densities of lectins, in turn enabling the study of the interaction for a wide range of glycans surface density. Equilibrium dissociation constants of a few $\mathrm{nM}$ were extracted for multivalent glycan-lectin binding, mimicking the conditions of biological membranes, whereas hundreds of $\mathrm{nM}$ were observed at the lower glycan surface densities.

\section{Introduction}

The use of high-throughput microarrays is gaining increasing acceptance as a method for the screening of libraries of biomolecules, such as DNA, proteins, peptides and sugars [1-4].

Among the different classes of molecules that can be assayed in multiplex format by microarray technology, glycans are of utmost importance as their interaction with a variety of biological targets, including antibodies, proteins, viruses and cells is crucial in many biological processes [5]. The array format is also of value in the quest for unnatural lectin ligands, such as glycomimetic molecules, that can be used to antagonize the action of specific lectins in natural settings. Glycomimetic antagonists have been developed for a limited number of lectins, mostly by trial and error processes, supported by molecular modeling of ligands and/or of ligand:lectin complexes [610]. Such molecules find application as tools to interrogate the glycobiology of human lectins, which is still largely unknown in its molecular details, and can also be used as leads in the 
development of antibacterial, antiviral and anti-inflammatory drugs. Drug discovery programs based on glycomimetics are greatly facilitated by the availability of glycomimetic arrays, which can be interrogated with individual lectins to identify specific leads. Glycan arrays have become widely available through public initiatives, such as the consortium for functional glycomics, and other similar consortia in Europe and Japan, and many groups use them to identify natural targets of newly discovered sugar-binding proteins (lectins) and to assess their specificity.

In glycan microarray technology, the slide surface chemistry has significant impact on ligand presentation, background noise, spot size, morphology and reproducibility. All these factors influence lectin-carbohydrate recognition. One of the key issues in the context of a multiplex assay is the ability to immobilize a variety of different carbohydrates on the same surface, with proper spacing and orientation [11-13]. Mono- and oligosaccharides suitably immobilized on a solid surface can reach a high local concentration, miming the multivalent presentation of glycans on proteins or cell surfaces, hence yielding carbohydrate-protein interactions much stronger than those typically measured for a monovalent format $[14,15]$. In this context, a soft and biologically inert polymer coating providing adequate accessibility to the immobilized glycans and enabling to control their surface density would be highly beneficial.

Cu-catalyzed azide/alkyne cycloaddition (CuAAC, click chemistry) has received a great deal of attention since its discovery, especially within glycobiology where several examples of carbohydrate arrays utilizing CuAAC have been published [16-21]. Most of the examples refer to 'clickable' self-assembled monolayers (SAMs) on glass and gold substrates $[20,21]$ whereas only a few examples of polymer-modified microarray slides allowing $\mathrm{Cu}(\mathrm{I})$-catalyzed azide-alkyne cycloaddition of probes have been reported [22-25] and none of them has been used in glycan microarray technology.

Herein we present a one-step method to functionalize glassy surfaces with alkynes using a polymer that produces a soft three-dimensional (3D) coating by a facile 'dip and rinse' method. The alkyne groups can subsequently be linked to azide-containing carbohydrates using Cu-catalyzed 
azide/alkyne cycloaddition (CuAAC, click chemistry) [26,27]. Both azides and alkynes are rare in biological systems and have been shown to be highly inert under biological conditions [28]. The advantages of a high quality click chemistry immobilization based on a 3D polymer coating are combined with the high fluorescence sensitivity and superior signal-to-noise ratio of a $\mathrm{Si}-\mathrm{SiO}_{2}$ substrate. The proposed approach enables the attachment of complex sugars on a silicon oxide surface via click chemistry by a method that does not require skilled personnel and chemistry laboratories.

Eight $\alpha$-mannoside derivatives, immobilized on the polymer-modified substrate, were screened against the mannose-binding lectin Concanavalin A (Con A), using $\alpha$-mannose as the positive control and $\beta$-galactose as the negative control. The fluorescence array analysis showed specific interactions of the mannosylated support with ConA with a high signal-to-noise ratio. The same surface immobilization strategy was also used on the sensing surface of the Reflective Phantom Interface (RPI) analytical platform [29]. This recently proposed optical label-free technique enables real-time monitoring of surface interactions; hence it is suited for the determination of binding constants and kinetics parameters. The detection principle is based on the simple measurement of the intensity of light reflected from a surface with very low reflectivity. At the highest surface densities of mannose derivatives, dissociation constants on the order of $1 \mathrm{nM}$ were calculated from both the fluorescence microarray experiments and the RPI method. The equilibrium dissociation constant $\left(\mathrm{K}_{\mathrm{D}}\right)$ of the interaction was found to strongly depend on the surface concentration of glycans. The fluorescence detection enhanced by the $\mathrm{Si} / \mathrm{SiO}_{2}$ substrates enabled to investigate binding properties of Concanavalin A at low glycan density and to determine surface equilibrium constants in solution-like conditions. 


\section{Materials and methods}

\subsection{Materials}

Trimethylsilylpropyn-1-ol, triethylamine (TEA), diethyl ether $\left(\mathrm{Et}_{2} \mathrm{O}\right)$, methacryloyl chloride $\left(\mathrm{CH}_{2} \mathrm{CCH}_{3} \mathrm{COCl}\right)$, dry tetrahydrofuran (THF), $\alpha, \alpha^{\prime}$-azoisobutyronitrile (AIBN), petroleum ether (EtP), potassium carbonate $\left(\mathrm{K}_{2} \mathrm{CO}_{3}\right)$, copper sulphate penta-hydrate $\left(\mathrm{Cu}_{2} \mathrm{SO}_{4} \cdot 5 \mathrm{H}_{2} \mathrm{O}\right)$, ascorbic acid, biotinylated ConcanavalinA (ConA), streptavidin-cyanine3, phosphate saline buffer (PBS), Bovin Serum Albumin (BSA), trizma base (Tris), chloridric acid ( $\mathrm{HCl})$, sodium chloride $(\mathrm{NaCl})$, Tween 20, manganese chloride $\left(\mathrm{MnCl}_{2}\right)$, calcium chloride $\left(\mathrm{CaCl}_{2}\right)$, sodium hydroxide $(\mathrm{NaOH})$, $\mathrm{N}-(2$ hydroxyethyl)piperazine-N'-(2-ethanesulfonic acid) (HEPES) were purchased from Sigma Aldrich (St. Louis , MO, USA). Cyanine3 azide was purchased from Lumiprobe GmbH (Feodor-Lynnen Strasse 23, 30625 Hannover, Germany). All solvents were used as received.

Silicon oxide chips with a $100 \mathrm{~nm}$ thermal oxide layer were bought from Silicon Valley Microelectronics (Santa Clara, CA, USA). The glass substrates with a silicon dioxide anti-reflection layer used in the RPI experiments were provided by ODL S.r.l. (Brembate Sopra, Bergamo, Italy). An Agilent 1200 series liquid chromatography system, (Agilent Technologies, Santa Clara, CA, USA) was used to carry out GCP. GPC columns were from Schodex (New York, NY, USA); MALLS system was purchased from Wyatt Technology (Santa Barbara, CA, USA).

\subsection{Polymer synthesis}

\subsubsection{Synthesis of 3-trimethylsilyl-prop-2-ynyl methacrylate (PMA)}

According to Ladmiral V. and co-workers [30] 3-(trimethylsilyl)prop-2-yn-1-ol (2.31 ml, 15.6 mmol) and triethylamine $(2.83 \mathrm{ml}, 20.27 \mathrm{mmol})$ were dissolved in $\mathrm{Et}_{2} \mathrm{O}(20 \mathrm{ml})$ and cooled to $20^{\circ} \mathrm{C}$. A solution of methacryloyl chloride $(1.81 \mathrm{ml}, 18.56 \mathrm{mmol})$ in $\mathrm{Et}_{2} \mathrm{O}(10 \mathrm{ml})$ was added drop wise over 1 hour. The mixture was stirred at $-20^{\circ} \mathrm{C}$ for 30 minutes and then overnight at room temperature. Ammonium salts were removed by filtration and the volatiles were removed under 
reduced pressure. The yellow oil residue was purified by flash chromatography (EtP:Et ${ }_{2} \mathrm{O}=50: 1$, $\mathrm{Rf}=0.39)(2.48 \mathrm{~g}, 12.64 \mathrm{mmol}$, Yield 81\%).

${ }^{1} \mathbf{H}-\mathrm{NMR}\left(400 \mathrm{MHz}, \mathrm{CDCl}_{3}\right): \delta=0.18\left(\mathrm{~s}, 9 \mathrm{H}, \mathrm{Si}\left(\mathrm{CH}_{3}\right)_{3}\right) ; 1.97\left(\mathrm{~m}, 3 \mathrm{H}, \mathrm{CH}_{3} \mathrm{C}=\mathrm{CH}_{2}\right) ; 4.76(\mathrm{~s}, 2 \mathrm{H}$, $\left.\mathrm{OCH}_{2}\right) ; 5.62(\mathrm{~m}, 1 \mathrm{H}, \mathrm{C}=\mathrm{CHH}) ; 6.17(\mathrm{~m}, 1 \mathrm{H}, \mathrm{C}=\mathrm{CH} H)$.

\subsubsection{Synthesis of copoly(N,N-dimethylacrylamide (DMA)- 3-trimethylsilyl-prop-2-ynyl} methacrylate (PMA)- 3-(Trimethoxysilyl)propyl methacrylate (MAPS)).

The polymer was synthesized via a random radical polymerization in anhydrous tetrahydrofuran with a $20 \% \mathrm{w} / \mathrm{v}$ total monomer concentration. The DMA was filtered on aluminium oxide to remove the inhibitor. The molar fraction of the monomers DMA, PMA and MAPS was 97:2:1.

The DMA and PMA monomers were dissolved in dried tetrahydrofuran (THF) in a round-bottom flask equipped with condenser, magnetic stirring. The solution was degassed by alternating argon purges with a vacuum connection, over a 10-min period. MAPS and $\alpha, \alpha^{\prime}$-Azoisobutyronitrile (this latter at $2 \mathrm{mM}$ final concentration) were added to the solution, which was then warmed to $65^{\circ} \mathrm{C}$ and maintained at this temperature under a slightly positive pressure of argon for $2 \mathrm{~h}$.

After the polymerization was completed, the solution was first diluted to $10 \% \mathrm{w} / \mathrm{v}$ with dry THF and the polymer precipitated by adding petroleum ether (10 times the reaction volume). The product, a white powder, was filtered on Buckner funnel and dried under vacuum at room temperature.

The protective trimethylsilyl groups were removed in water under basic condition, using $\mathrm{K}_{2} \mathrm{CO}_{3}$ $(9 \mathrm{mM})$ at $\mathrm{pH}$ 9. The reaction mixture was stirred at room temperature for $1 \mathrm{~h}$, then the polymer was dialyzed, lyophilized and the white powder obtained was stored at $-20{ }^{\circ} \mathrm{C}$.

\subsubsection{Polymer characterization by Gel Permeation Chromatography}

The size of each polymer was characterized using Gel Permeation Chromatography in tandem with an UV-detector $(\lambda=214 \mathrm{~nm})$. 
A JASCO 880 PU liquid chromatography system, consisting of an isocratic pump to control mobile phase flow throughout the system connected to a JASCO UVIDEC-100-III UV detector. ChromNAV Chromatography Data System -JASCO was used to analyze the sequence of sample injection and to calculate the calibration curve of polyacrylamide standards.

The GPC setup consists of four Shodex aqueous GPC columns in series: OHpak SB-G (guard column), OHpak SB-804M HQ, OHpak SB-803 HQ, and OHpak SB-802.5 HQ. Each column is packed with a polyhydroxymethacrylate gel and connected in series with a decreasing exclusion limit. The columns were maintained at $40^{\circ} \mathrm{C}$ throughout each run using a thermostated column compartment.

After the polymer sample is fractionated by GPC, the sample flows into a UV-detector. The molecular weight of the polymer was obtained by using a calibration curve.

Copoly(DMA-PMA-MAPS) sample was diluted using the GPC mobile phase (GPC buffer: 100 $\mathrm{mM} \mathrm{NaCl}, 50 \mathrm{mM} \mathrm{NaH}_{2} \mathrm{PO}_{4}, \mathrm{pH} 3,10 \% \mathrm{v} / \mathrm{v}$ Acetonitrile) to a concentration of $2.66 \mathrm{mg} / \mathrm{ml}$ and the sample was run three times through the GPC-UV system to test for reproducibility. Each run injected $20 \mu \mathrm{L}$ of sample to be analyzed and the flow rate through the system was held at a constant $0.3 \mathrm{~mL} / \mathrm{min}$.

\subsection{Goniometry}

Contact angle measurements were collected via the sessile drop method using a CAM200 instrument (KSV Ltd), which utilizes video capture and subsequent image analysis. Deionized water was used, and its purity was confirmed by correlating the measured surface tension based on the pendant drop shape to the literature values for pure water $\left(72 \mathrm{mN} / \mathrm{m}\right.$ at $\left.25^{\circ} \mathrm{C}\right)$.

\subsection{Dual Polarization Interferometry (DPI)}

Dual polarization interferometry (DPI) measurements were conducted using an Analight Bio 200 (Farfield Group, Manchester, UK) running Analight Explorer software. 


\subsection{Fluorescence glycan microarray experiments}

Four hundreds pL of each glycan were spotted at various concentrations as detailed in the text from an aqueous solution of $\mathrm{Cu}_{2} \mathrm{SO}_{4} \cdot 5 \mathrm{H}_{2} \mathrm{O}(2.5 \mathrm{mM})$ and ascorbic acid $(12.5 \mathrm{mM})$ with a piezoelectric spotter (SciFlexArrayer S5, Scienion, Berlin Germany). The immobilization reaction took place during an overnight incubation in a humid chamber at room temperature. The printed slides were sequentially washed with PBS buffer for 10 minutes with DI water and dried by a nitrogen stream. The arrayed slides were then incubated with biotinylated $\alpha$-mannose-binding lectin Concanavalin A (ConA) in the lectin binding buffer (LBB, $50 \mathrm{mM}$ HEPES, pH 7.4, 5mM $\mathrm{MnCl}_{2}, 5 \mathrm{mM} \mathrm{CaCl}$ ) in the presence of BSA $(0.2 \mathrm{mg} / \mathrm{ml})$. After 2 hours of incubation at room temperature on a lab shaker, the slides were washed 10 minutes in washing Buffer (0.05 M Tris/ $\mathrm{HCl}$ pH9, $0.25 \mathrm{M} \mathrm{NaCl,} \mathrm{0.05 \%}$ v/v Tween 20), rinsed in DI water and dried by a nitrogen stream. A final incubation of $1 \mathrm{~h}$ with $2 \mu \mathrm{g} / \mathrm{ml}$ Cyanine3 labelled Streptavidin in PBS (Phosphate Saline Buffer) in a humid chamber at room temperature under static condition enabled the fluorescence detection of the surface bound ConA by means of a scanner (ProScanArray scanner from Perkin Elmer, Boston, MA, USA) used at $70 \%$ of laser power and $60 \%$ of photomultiplier (PMT) gain. The fluorescence intensities of 11 spot replicates were confirmed by three experiments that provided the same fluorescence intensities for each glycomimetic, with a standard deviation lower than 5\%. The experimental conditions used during the incubation were optimized to ensure attainment of the equilibrium.

\subsection{Reflective phantom interface (RPI) experiments}

The affinity and the kinetics of the interaction between two mannose derivatives and ConA were characterized by Reflective Phantom Interface (RPI), an optical label-free method, whose theoretical and technical details are given elsewhere [29,31]. A glass prism with an antireflection layer, functionalized with the copolymer and glycans 9 and 10, was placed into a cuvette containing a magnetic stir bar and the sensing surface was illuminated by the light of a LED at $595 \mathrm{~nm}$. The image of the reflected light was acquired by a CCD camera, while increasing concentrations of 
biotinylated ConA were added in a $10 \mathrm{mM}$ Hepes buffer at pH 7.4, containing $2 \mathrm{mM} \mathrm{CaCl}_{2}, 2 \mathrm{mM}$ $\mathrm{MnCl}_{2}, 150 \mathrm{mM} \mathrm{NaCl}$ and $0.2 \mathrm{mg} / \mathrm{ml} \mathrm{BSA}$ at room temperature. The brightness $u(t)$ of each spot as a function of time was converted into the normalized surface density of target molecules $\square=$ $\left(u(t) / u_{0}-1\right)^{1 / 2}$, where $u_{0}$ is the brightness of the bare chip surface. The binding curves obtained at different concentrations $c$ of ConA were fitted with exponential functions, whose characteristic rates, $\square(c)$, and amplitudes, $A(c)$, were used to extract the values of the dissociation $\left(\mathrm{k}_{\mathrm{off}}\right)$ and association $\left(\mathrm{k}_{\mathrm{on}}\right)$ kinetic constants and of $\mathrm{K}_{\mathrm{D}}=\mathrm{k}_{\text {off }} / \mathrm{k}_{\mathrm{on}}$, being $\square=\mathrm{k}_{\text {on }} c+\mathrm{k}_{\text {off }}$ and $A=A_{0} /\left(1+\mathrm{K}_{\mathrm{D}}\right.$ $/ c)$

\section{Results and discussion}

\subsection{Design of the polymer structure and substrate selection}

The performance of a solid phase sensor is dictated by a number of factors of which the most important are: the coupling efficiency of the analyte to the surface, the strength of recognition, and the method of detection. The microarray technology proposed by this work brings about significant improvements to these three factors, by exploiting a novel 3D, soft functional coating on $\mathrm{Si} / \mathrm{SiO}_{2}$ slides.

Hydrophilic 3D coatings have found wide application in solid phase assays due to their ability to maximize the probe loading capacity and to simulate an aqueous environment [32]. Furthermore, they can mitigate the steric effects that alter the access of carbohydrate ligand to the protein binding pockets, spacing the targets from the surface and conferring to the interface mechanical properties similar to those of soft biological tissues.

Concerning the method of detection, by replacing glass with $\mathrm{Si} / \mathrm{SiO}_{2}$, slides a strong fluorescence enhancement is obtained. As previously shown, the optical interference (OI) phenomenon induced by layers of well-defined thickness and different refractive index, maximizes photo-absorption of the dye molecules in the vicinity of the surface and enhances the light emitted towards the detector [33]. The $\mathrm{Si} / \mathrm{SiO}_{2}$ microarray slides display fluorescence intensity 4 times higher than that of 
standard glass slides (See Figure 1S).

The polymer coating introduced in this work, named copoly(DMA-PMA-MAPS), is obtained from the polymerization of N,N-dimethylacrylamide (DMA), 3-trimethylsilanyl-prop-2-yn methacrylate (PMA) and 3(trimethoxysilyl)-propylmethacrylate (MAPS) (Figure 1). The GPC-MALLS analysis of copoly(DMA-PMA-MAPS) indicates that the polymer has a molecular weight (Mw) of $4.2 \times 10^{4}$ $\mathrm{g} / \mathrm{mol}$ and polydispersity of 2.6. This new copolymer is based upon a copolymer introduced by our group to form a hydrophilic 3D coating for microarray [34] on a variety of materials. As a general rule, in order to perform as an efficient 3D coating array substrate the copolymer must have 1) a segment that interacts with the surface by weak, non covalent interactions such as hydrogen bonding, Van der Waals or hydrophobic forces (polymer backbone), 2) a pending hydrolysable silane functionality to promote condensation of the polymer with surface silanols and 3) chemically active monomers whose reactivity is selected on the basis of the reactivity of the molecules that have to be immobilized. In this work the reactive monomer bears an alkyne moiety, to allow immobilization of azide-modified glycans by azide alkyne Huisgen cycloaddition using a Copper (Cu) catalyst at room temperature (Figure 2). Binding glycans to the surface via click chemistry offers a number of advantages [22,35,36] over classical nucleophilic reactions between amino modified probes and surface-active esters. From the surface point of view, the stability of an alkyne group is far higher than that of an active ester, which typically needs to be freshly prepared right before the immobilization reaction. Additionally, when building arrays of natural glycans, the selectivity of the attachment point is guaranteed, as there are no natural glycans that contain azido functions. Last but not least, when building microarrays of glycomimetics for medicinal chemistry purposes, the chemoselectivity of the Huisgens cycloaddition is more tolerant of functional groups that can be explored in the mimics. The molar fraction of the three monomers and the coating protocol have been optimized starting from the conditions initially used with the parent copoly(DMA-NAS-MAPS). The polymer, as powder, is stable for one year whereas its coating solutions must be used within few hours. Replacing NAS with PMA does not alter either the self- 
adsorbing properties of the polymer or its physical characteristics, therefore polymer concentration, additives and temperatures used in the coating process were similar to those optimized for the parent copolymer (DMA-NAS-MAPS) in previous works [31-34]. The coating process requires immersing the slides in a $1 \% \mathrm{w} / \mathrm{v}$ solution of copoly(DMA-PMA-MAPS) in $0.8 \mathrm{M}$ ammounium sulphate for 30 minutes. Following incubation with the coating solution, the slides were rinsed in DI water, dried with nitrogen flow and then cured at $80^{\circ} \mathrm{C}$ under vacuum for 15 minutes. Before the coating, the surface was pre-treated for 10 min with oxygen plasma in a Plasma Cleaner from Harrick Plasma (Ithaca, NY, USA) with the oxygen pressure set at 1.2 Bar and the power at 29.6 W. The film was characterized by contact angle measurements both before and immediately after the coating deposition. Significant changes of the surface hydrophilicity resulting from the presence of a surface polymer layer were observed. The water contact angle could not be measured on an uncoated silicon chip after 10 minutes of plasma oxygen treatment because of its extremely high hydrophilicity (i.e. complete wetting). Thanks to this characteristic, the formation of a polymer coating is immediately evident because the water droplet contact angles increase on the coated surfaces from $0^{\circ}$ to $33^{\circ} \pm 0.78{ }^{\circ} \mathrm{C}$ (the obtained contact angle value is the average of five measurements on five different chips).

The physical parameters of the coating were characterized using dual polarization interferometry (DPI) [37]. DPI is currently one of the most powerful label-free biosensing techniques in heterogeneous format that allows measuring refractive index and thickness values of a thin film almost simultaneously by measuring two different interference fringe patterns. These patterns can be mathematically resolved into refractive index and thickness values, and hence, the final outcome is a measurement in real time of both parameters. In this technique, a silicon chip (AnaChip ${ }^{\mathrm{TM}}$ ) with an oxynitride surface treated with oxygen plasma was inserted into the fluidic compartment of an Analight Bio 200 (Farfiled, UK) and a polymer solution (1\% w/v in a 0.8M ammonium sulphate solution ) was slowly introduced at a flow rate of $6 \mu 1 / \mathrm{min}$ for a total time of 15 minutes into the channels of the chip. The flow was then stopped, and the solution was let in contact with the surface 
for 30 minutes before washing the channel with water at a flow rate of $50 \mu \mathrm{l} / \mathrm{min}$. As shown in Table 1, the polymer layer has a thickness of about $15 \mathrm{~nm}$ and it is massively hydrated as indicated by its low density.

The saturation density of immobilization sites on the polymer was estimated measuring the fluorescence after spotting, immobilization and washing of an azide-modified Cyanine-3 dye (1, Figure 3), following a procedure described by Liang et al [38]. A value of about 3 molecules $/ \mathrm{nm}^{2}$ is obtained for printing concentration higher than $500 \mu \mathrm{M}$ (ESI, Figure 3S). The surface density of the molecules immobilized in a spot shows an approximate linear dependence with the dye concentration in the spotting solution, as reported in Figure 4. The enhancement of fluorescence sensitivity provided by the $\mathrm{Si} / \mathrm{SiO}_{2}$ slides enables the detection with high signal-to-noise ratio of rather low amounts of dyes onto the surface. Less than 0.05 molecules $/ \mathrm{nm}^{2}$ are clearly imaged with the commercial scanner employed in this study, corresponding to a printing concentration of $0.5 \mu \mathrm{M}$ of Cy3 dye.

\subsection{Determination of the equilibrium dissociation constant $\left(K_{D}\right)$}

The eight $\alpha$-mannose derivatives $\mathbf{2 - 9}$ shown in Figure 3 were spotted on the surface of a polymer coated $\mathrm{Si} / \mathrm{SiO}_{2}$ slide at $50 \mu \mathrm{M}$ concentration. $\alpha$-mannose (10) and $\beta$-galactose (11) were used as positive and negative controls, respectively, whereas the Cy3 derivative was used as a reference to facilitate the imaging process. Concanavalin A (ConA) was chosen in this work, due to its well characterized affinity for mannose and glucose derivatives [39, 40].

The surface-immobilized glycans, incubated with $100 \mathrm{ng} / \mathrm{ml}(0.943 \mathrm{nM})$ of biotinylated ConA and detected with Cy3-labelled streptavidin, show a variable degree of fluorescent intensity (Figure 5a) depending on their affinity for ConA. The interaction between $\alpha$-mannose derivatives and ConA was specific as confirmed by the lack of fluorescence on the spots of $\beta$-galactose (11), the negative control. Spotted slides are stable for months when stored in a dry environment. The graph of Figure 5b reports the fluorescence intensity observed for different glycan spots. Except for ligand 5, all the 
mannosides of this study as well as the control $\mathbf{1 0}$ have similar affinities for ConA, as expected from their strong structural similarities. On the contrary, ligand $\mathbf{5}$ does not seem to interact, possibly due to steric hindrance from the large, lipophilic amide groups. The analysis reported above provided only a qualitative estimate of the affinity between the $\alpha$-mannose derivatives immobilized onto the surface and the selected lectin. In order to measure the equilibrium dissociation constant $\left(\mathrm{K}_{\mathrm{D}}\right)$ of the interaction a more complex experiment was required. According to a method previously reported by Liang and co-workers [38], nine slides were spotted with $50 \mu \mathrm{M}$ and $10 \mu \mathrm{M}$ aqueous solutions of 11 replicates of the glycomimetics 2-11 (Figure 3). The chips were incubated with ConA solutions of increasing concentration, from $0.05 \mathrm{nM}$ up to $9.4 \mathrm{nM}$. For each glycan, average values of fluorescence were plotted against ConA concentrations (logarithmic scale) and the curve was fitted as a sigmoidal/growth function using OriginPro-8 fixing the parameter $\mathrm{p}=1$ and the parameter $\mathrm{A} 1=0$. Typical curves of high (3) and low affinity (5) glycomimetics are shown in Figure 4S (See ESI). The values of the equilibrium dissociation constant $K_{D}$ extracted from the fit, reported in Table 2, provide a quantitative estimation of the affinity between the glycomimetics and ConA, when the interaction occurs on a surface at a given ligand density. These values are considerably lower than those reported in the literature for interactions taking place in solution [41], but consistent with values determined by other authors based on surface interactions [38]. The observed discrepancies between the values of $K_{D}$ obtained in solution or on surface could be ascribed to the high local concentration of glycans that are likely to behave like cell-surface glycoconjugates [42, 43] and bind lectins through multiple, simultaneous interactions. Others have reported this effect in different experimental conditions $[44,45]$. ConA is

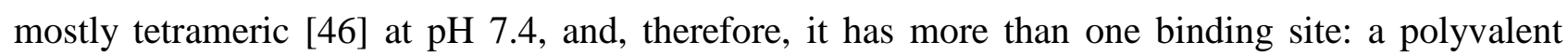
presentation of the glycans is required to activate the intrinsic avidity of the lectin (Table 3).

\subsection{Effect of surface density of glycans}


The enhanced sensitivity of the array slides proposed here enables to investigate the effect of probe density on ligand avidity [34]. Unlike most glass slides [38], where the detection of fluorescence signals at low surface density is rather challenging, the sugar interaction with ConA could be evaluated on $\mathrm{Si} / \mathrm{SiO}_{2}$ slides in conditions were the glycans are spaced enough to mimic the monovalent interaction typical of an assay with free glycans in solution.

We investigated this matter using the $\alpha$ - mannoside (10) and the $\alpha$-mannose derivative (9) as probes. Both glycans were printed on a copoly(DMA-PMA-MAPS) coated surface at four different concentrations and incubated with a wide range of ConA solutions (from $0.450 \mathrm{nM}$ up to $13.4 \mu \mathrm{M}$ ). The density of probes immobilized in each spot as a function of their concentration in the spotting solution was assumed to be similar to that estimated using an azido-modified Cyanine 3 dye (details are reported in the Supplementary information file). In Figure 5S, typical dose response curves of fluorescence versus ConA concentrations at various glycan surface densities are depicted for $\mathbf{1 0}$. Similar curves were obtained for $\mathbf{9}$. By extrapolating $K_{D}$ from these curves, it is evident that as the glycan surface density decreases also the affinity decreases (see Table 3). However, in none of the experimental conditions used here we were able to reproduce values of $K_{D}$ higher than a few hundreds $\mathrm{nM}$, whereas values in the range $0.3-10 \mu \mathrm{M}$ were reported for competitive assay in solution [38], or using classical SPR analysis [47] with surface-immobilized lectins. The discrepancy of $K_{D}$ values determined in this work and by SPR highlights the importance of the surface environment on interactions taking place at the solid-liquid interface.

To confirm the increased avidity of glycans when immobilized on the proposed surface we have measured the strength and the kinetics of the interaction by an innovative label-free method [29] named Reflective Phantom Interface technology. The method enables quantifying the amount of target molecules bound to probes immobilized on a surface that has extremely low intrinsic reflectivity in an aqueous solution. The sensor, a glass chip with an anti-reflection layer of silicon dioxide, was coated with copoly(DMA-PMA-MAPS) and spotted with the mannose derivatives, similarly to the fluorescence microarray experiments. The choice of this platform instead of the 
more consolidated Surface Plasmon Resonance is motivated by the use of the exact same immobilization chemistry in the two different platforms which wouldn't be possible with SPR that requires a gold sensor. The glycans $\mathbf{9}$ and $\mathbf{1 0}$ were i $\mu$ mobilized on glass chip treated with antireflective $\mathrm{SiO}_{2}$ layer and coated by the polymer in spots with about $200 \mathrm{~mm}$ diameter obtained with a $5 \mathrm{mM}$ spotting solution. The addition of ConA in the buffer solution in contact with the surface in the measuring cuvette produced a real time increase of reflectivity related to the amount of protein binding to the mannose derivatives onto the surface. Figure 6a [48] shows the time dependent increase of the normalized surface density on top of the spots, while the concentration of ConA is brought from 0 to about $50 \mathrm{nM}$. The amplitudes and the characteristic times of each binding curve were extracted from the fit with exponential growth functions and plotted as a function of the concentration in Figure 6b. From their fit, the values of the kinetic dissociation constant $\mathrm{k}_{\text {off }}$ and the value of $K_{D}=K_{o f f} / k_{\text {on }}$ were obtained, yielding very similar values for the two glycans: $K_{D}=4.9$ $\pm 0.7 \mathrm{nM}$ and $\mathrm{k}_{\text {off }}=1.88 \pm 0.410^{-4} \mathrm{~s}^{-1}$ for $\mathbf{9}$ and $\mathrm{K}_{\mathrm{D}}=4.5 \pm 0.7 \mathrm{nM}$ and $\mathrm{k}_{\text {off }}=1.34 \pm 0.510^{-4} \mathrm{~s}^{-1}$ for $\mathbf{1 0}$. Equilibrium dissociation constants measured by RPI and by microarray technology are similar and both indicates a much stronger interaction than those obtained in other approaches relying on monovalent glycan-lectin binding [38], [48].

\section{Conclusions}

In this work we introduced a new polymer obtained from the polymerization of $\mathrm{N}, \mathrm{N}$ dimethylacrylamide (DMA), 3-trimethylsilanyl-prop-2-yn methacrylate (PMA) and 3(trimethoxysilyl)-propylmethacrylate (MAPS), copoly(DMA-PMA-MAPS) and describe its use in the formation of a functional 3D, soft coating for microarrays. The backbone of the polymer bears alkyne moieties that allow binding azide-modified glycans to the surface by "Click" chemistry. This attachment mode offers a number of advantages in the immobilization of glycans, such as high grafting efficiency, oriented immobilization and insensitivity to functionalities present in natural 
glycans. The novel surface chemistry was used to prepare microarrays substrates for two different analytical platforms: fluorescence microarray on $\mathrm{Si} / \mathrm{SiO}_{2}$ slides and RPI label-free detection. The strength and the kinetics of the interaction between the surface immobilized glycans and ConA in solution were cross-validated on the two platforms, which provided similar values. The higher sensitivity to the fluorescence signal provided by the novel $\mathrm{Si} / \mathrm{SiO}_{2}$ microarray substrate offers significant advantages over conventional glass slides allowing analysis at lower glycan surface density. 


\section{ACKNOWLEDGEMENT}

Financial support from EU FP7 NADINE (Nanosystems for Early Diagnosis of Neurodegenerative Diseases) is gratefully acknowledged. We would like to thank ODL S.r.l.for providing the Glass substrate for the RPI Method.

\section{References}

[1] D. Castel, A. Pitaval, M. Debily, and X.Gidrol, Cell microarrays in drug discovery, Drug Discov. Today, 11 (2006) 616-622.

[2] A. Schulze, and J. Downward, Navigating gene expression using microarrays - a technology review, Nature Cell Biol. 3 (2001) 190-195.

[3] G. Ramsay, DNA chips: state-of-the art, Nature Biotechnol. 16 (1998) 40-44.

[4] D. Anderson, D. Putnam, E. B. Lavik, T. A. Mahmood, R. Langer, Biomaterial microarrays: rapid, microscale screening of polymer-cell interaction, Biomaterials 26 (2005) 4892-4897.

[5] C.Y. Wu, P.H. Liang and C.H. Wong, New development of glycan arrays, Org. Biomol. Chem. 7 (2009) 2247-2254.

[6] H. C. Kolb, B. Ernst, Development of Tools for the Design of Selectin Antagonists, Chem. Eur. J. 3 (1997) 1571-1578.

[7] N. Kaila, B. E. Thomas, Design and synthesis of sialyl Lewis ${ }^{\mathrm{x}}$ mimics as E- and P-selectin inhibitors, IV Med. Res. Rev. 22 (2002) 566-601.

[8] B. Ernst and J. L. Magnani, From carbohydrate leads to glycomimetic drugs, Nat. Rev. Drug Discov., 8 (2009) 661-677.

[9] L.R. Prost, J.C. Grim, M. Tonelli, and L.L. Kiessling, Noncarbohydrate Glycomimetics and Glycoprotein Surrogates as DC-SIGN Antagonists and Agonists, ACS Chem. Biol. 7 (2012) 16031608.

[10] A. Bernardi, P. Cheshev, Interfering with the Sugar Code: Design and Synthesis of Oligosaccharide Mimics, Chem. Eur. J. 14 (2008) 7434-7441. 
[11] O. Oyelaran, J. C. Gildersleeve, Glycan arrays: recent advances and future challenges, Curr. Opin. Chem. Biol. 13 (2009) 406-413.

[12] C.D. Rillahan and J.C. Paulson, Glycan microarrays for decoding the glycome, Annu. Rev. Biochem. 80 (2011) 797-823.

[13] W. Peng, C. M. Nycholat, N. Razi, Glycan microarray screening assay for glycosyltransferase specificities, Methods Mol. Biol. 1022 (2013) 1-14.

[14] Y. Zhang, Q. Li and J. C. Gildersleeve, An Array-Based Method to Identify Multivalent Inhibitors, J. Am. Chem. Soc. 132 (2010) 9653-9662.

[15] O. Oyelaran, Q. Li and J. C. Gildersleeve, Microarrays with Varying Carbohydrate Density Reveal Distinct Subpopulations of Serum Antibodies, J. Proteom Res. 8 (2009) 3529-3538.

[16] S. R. Hanson, W. A. Greenberg, C-H. Wong, Probing Glycans With the Copper(I)-Catalyzed [3+2] Azide-Alkyne Cycloaddition., QSAR Comb. Sci. 26 (2007) 1243-1252.

[17] F. Fazio, M.C. Bryan, O. Blixt, J.C. Paulson, C.-H. Wong, Synthesis of Sugar Arrays in Microtiter Plate, J. Am. Chem. Soc. 124 (2002) 14397-14402.

[18] M.C. Bryan, F. Fazio, H.-K. Lee, C.-Y. Huang, A. Chang, M.D. Best, D.A. Calarese, O. Blixt, J.C. Paulson, D. Burton, I.A. Wilson, C.-H. Wong, Covalent Display of Oligosaccharide Arrays in Microtiter Plates, J. Am. Chem. Soc. 126 (2004) 8640-8641.

[19] P. Wu, M. Malkoch, J.N. Hunt, R. Vestberg, E. Kaltgrad, M.G. Finn, V.V. Fokin, K.B. Sharpless, C.J. Hawker, Multivalent bifunctional dendrimers prepared by click chemistry., Chem. Commun. 46 (2005) 5775-5777.

[20] X.-L. Sun, C.L. Stabler, C.S. Cazalis, E.L. Chaikof, Carbohydrate and Protein Immobilization onto Solid Surfaces by Sequential Diels-Alder and Azide-Alkyne Cycloadditions, Bioconjugate Chem. 17 (2006) 52-57.

[21] Y. Zhang, S. Luo, Y. Tang, L. Yu, K.-Y. Hou, J.-P. Cheng, X. Zeng, P.G. Wang, Carbohydrate-Protein Interactions by "Clicked" Carbohydrate Self-Assembled Monolayers, Anal. Chem. 78 (2006) 2001-2008.

[22] O. Michel, B.J. Ravoo, Carbohydrate Microarrays by Microcontact "Click" Chemistry, Langmuir 24 (2008) 12116-12118.

[23] N. Himabindu, H.-Y. Chen, L. Bondarenko and J. Lahann, Reactive polymers coating that “Click“, Angew. Chem. Int. Ed. 45 (2006) 3360-3363.

[24] W.J. Yang WJ, D. Pranantyo, K.G. Neoh, E.T. Kang, S.L. Teo, D. Rittschof, Layer-by-layer click deposition of functional polymer coatings for combating marine biofouling, Biomacromolecules 13 (2012) 2769-2780. 
[25] S. Saha , M.L. Bruening *, and G.L. Baker, Surface-Initiated Polymerization of Azidopropyl Methacrylate and its Film Elaboration via Click Chemistry, Macromolecules 22 (2012), 9063-9069 [26] V.V. Rostovtsev, L.G. Green, V.V. Fokin, K.B. Sharpless, A stepwise Huisgen cycloaddition process: copper(I)-catalyzed regioselective "ligation" of azides and terminal alkynes, Angew.

Chem. Int. Ed. 41 (2002) 2596-2599.

[27] C.W. Tornoe, C. Christensen, M. Meldal, Peptidotriazoles on Solid Phase: [1,2,3]-Triazoles by Regiospecific Copper(I)-Catalyzed 1,3-Dipolar Cycloadditions of Terminal Alkynes to Azides, J. Org. Chem. 67 (2002) 3057-3064.

[28] Q. Wang, T.R. Chan, R. Hilgraf, V.V. Fokin, K.B. Sharpless, M.G. Finn, Bioconjugation by Copper(I)-Catalyzed Azide-Alkyne [3 + 2] Cycloaddition, J. Am. Chem. Soc. 125 (2003) 31923193.

[29] F. Giavazzi, M., Salina, R. Cerbino, M Bassi, D. Prosperi, E. Ceccarello, F. Damin, L. Sola, M. Rusnati, M. Chiari, B. Chini, T. Bellini, M. Buscaglia, Multispot label-free biodetection at a phantom plastic-water interface, P. Natl. Acad. Sci. USA, 110 (2013) 9350-9355.

[30] V. Ladmiral, G. Mantovani, G. J. Clarkson, S. Cauet, J. L. Irwin, and D. M. Haddleton, Synthesis of Neoglycopolymers by a Combination of “Click Chemistry” and Living Radical Polymerization, J. Am. Chem. Soc. 128 (2006) 4823-4830.

[31] F. Giavazzi, M. Salina, E. Ceccarello, A. Ilacqua, F. Damin, L. Sola, M. Chiari, B. Chini, R. Cerbino, T. Bellini, M. Buscaglia, A fast and simple label-free immunoassay based on a smartphone, Biosens. Bioelectron., 58 (2014) 395-402.

[32] M. Cretich, G. Di Carlo, R. Longhi, C. Gotti, N. Spinella, C. Galati, L. Renna, M. Chiari, High sensitivity protein assays in microarray silicon slides. Anal. Chem., 81 (2009) 5197-5203.

[33] M. Cretich, A. Reddington, M. Monroe, M. Bagnati, F. Damin, L. Sola, M. S. Unlu, M. Chiari, Silicon biochips for dual label/free and fluorescence detection: application to protein microarrays development, Biosens. Bioelectron. 26 (2011) 3938-3943.

[34] G. Pirri, F. Damin, M. Chiari, E. Bontempi and L. E. Depero, Characterization of a polymeric adsorbed coating for DNA microarray glass slides, Anal. Chem. 76 (2004) 1352-1358.

[35] S. Bian, J. He, K. B. Schesing, A. B. Braunschweig, Polymer Pen Lithography (PPL)-Induced Site-Specific Click Chemistry for the Formation of Functional Glycan Arrays, Small 8 (2012) 20002005.

[36] E. H. Song and N. L. B. Pohl, Carbohydrate arrays: recent developments in fabrication and detection methods with applications, Curr. Op. Chem. Biol. 13 (2009) 626-632.

[37] M. Swann, L. Peel, S. Carrington, N. Freeman, Dual Polarization Interferometry: an analytical technique to measure changes in protein structure in real time, to determine the stoichiometry of 
binding events, and to differentiate between specific and non-specific interactions, Anal. Biochem. 329 (2004) 190-198.

[38] P. Liang, S. Wang, and C. Wong, Quantitative Analysis of Carbohydrate-Protein Interactions Using Glycan Microarrays: Determination of Surface and Solution Dissociation Constants, J. Am. Chem. Soc. 129 (2007) 11177-11184.

[39] R. Loris, T. Hamelryck, J. Bouckaert, L. Wyns, Legume lectin structure, Biochim. Biophys. Act. 1383 (1998) 9-36.

[40] E. J. M. Van Damme, W. J. Peumans, A. Barre, P. Rougé, Plant lectins: a composite of several distinct families of structurally and evolutionary related proteins with diverse biological roles, Crit. Rev. Plant Sci. 17 (1998) 575-692.

[41] E. A. Smith, W. D. Thomas, L. L. Kiessling and R. M. Corn, Surface plasmon resonance imaging studies of protein-carbohydrate interactions, J. Am. Chem. Soc. 125 (2003) 6140-6148.

[42] R. Liang, L. Yan, J. Loebach et al., Parallel synthesis and screening of a solid phase carbohydrate library, Science 274 (1996) 1520-1522.

[43] R. Liang, J. Loebach, N. Horan et al., Polyvalent binding to carbohydrates immobilized on an insoluble resin, Proceedings of the National Academy of Sciences of the United States of America 94 (1997) 10554-10559.

[44] N. Horan, L. Yan, H. Isobe, G.M. Whitesides D.Kahne, Nonstatistical binding of a protein to clustered carbohydrated P. Natl. Acad. Sci. USA, 1999 96, 11782-11796.

[45] Y. C. Lee, R. T. Lee, Carbohydrate-Protein Interactions: Basis of Glycobiology, Acc. Chem. Res. 28 (1995) 321-327.

[46] D.F. Senear, D. C. Teller, Thermodynamics of concanavalin A dimer-tetramer self-association: sedimentation equilibrium studies, Biochem. 20 (1981) 3076-3083.

[47] R. Gutiérrez Gallego, S. R. Haseley, V. F. Van Miegem, J. F. Vliegenthart, J. P. Kamerling, Identification of carbohydrates binding to lectins by using surface plasmon resonance in combination with HPLC profiling, Glycobiology 14 (2004) 373-386.

[48] S. Swillens, Interpretation of binding curves obtained with high receptor concentrations: practical aid for computer analysis, Mol. Pharmacol., 47 (1995) 1197-1203.

\section{Legend}

Figure 1: Synthesis of the copoly(DMA-PMA-MAPS) copolymer. In brackets the molar fractions of the monomers.

Figure 2: Reaction scheme of a typical click reaction between the surface and the glycan. 
Figure 3: Chemical formula of azide cyanine dye (1), $\alpha$-mannose derivatives (2-9) positive (10, $\alpha$ mannose) and negative (11, $\beta$-galactose) controls.

Figure 4: Linear trend of the dye density $\left(\right.$ molecules $/ \mathrm{cm}^{2}$ ) as a function of its printing concentration $(\mu \mathrm{M})$

Figure 5: Mean fluorescence intensity of the glycomimetics of Table 1 (11 replicates per line) incubated with $100 \mathrm{ng} / \mathrm{ml}$ of biotynilated ConA (0.943 nM) and revealed with Cy3 labelled streptavidin, (a) image of the glycomimetic microarray; (b) histogram of spot fluorescence intensity of 11 spot replicates.

Figure 6: Characterization of glycan/ConA interactions by RPI. (a) The light reflected by spots of 9 (red curves), $\mathbf{1 0}$ (green curves) and $\mathbf{1 1}$ (blue curves) is measured as a function of time, while increasing concentrations of ConA are added to the buffer solution. The vertical dashed lines indicate the addition times, and the corresponding concentrations are reported in the figure. The reflection signal is converted into normalized surface density and fitted with single exponential growth functions (black lines). At the highest concentration (48.5 nM), a small linear contribution is added to the fit, in order to account for non-specific adhesion taking place with very low kinetics ${ }^{34}$. (b) The equilibrium plateaus of the binding curves reported in panel a are shown as a function of the ConA concentration and fitted with a sigmoidal curve (see Materials and Methods), from which the dissociation constant $K_{D}$ for the interaction is obtained. Inset: the rate of the exponentials reported in panel a are fitted with a linear function of the concentration, with the slope and the intercept represented by $\mathrm{k}_{\mathrm{on}}$ and $\mathrm{k}_{\mathrm{off}}$, respectively, and $\mathrm{k}_{\mathrm{off}} / \mathrm{k}_{\mathrm{on}}=\mathrm{K}_{\mathrm{D}}$. 
$\prod_{\text {PMA }}^{\text {OII }}$

(2\%)<smiles>C=C(C)C(=O)OCCC[Si](C)(O)OC</smiles>

MAPS

(1\%)

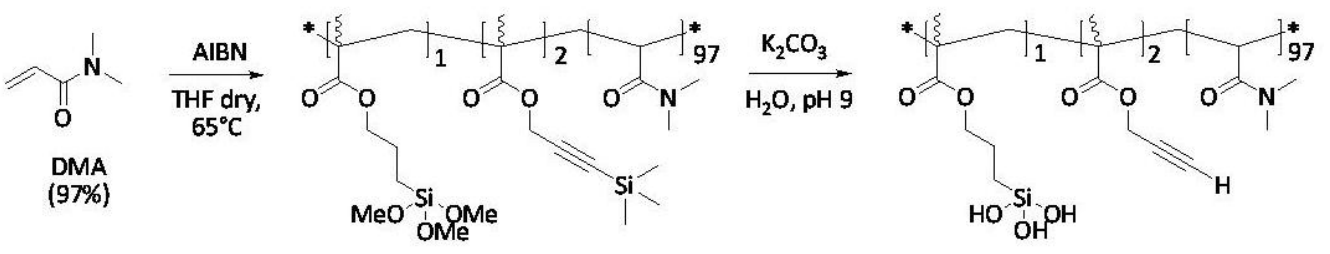

Figure 1 

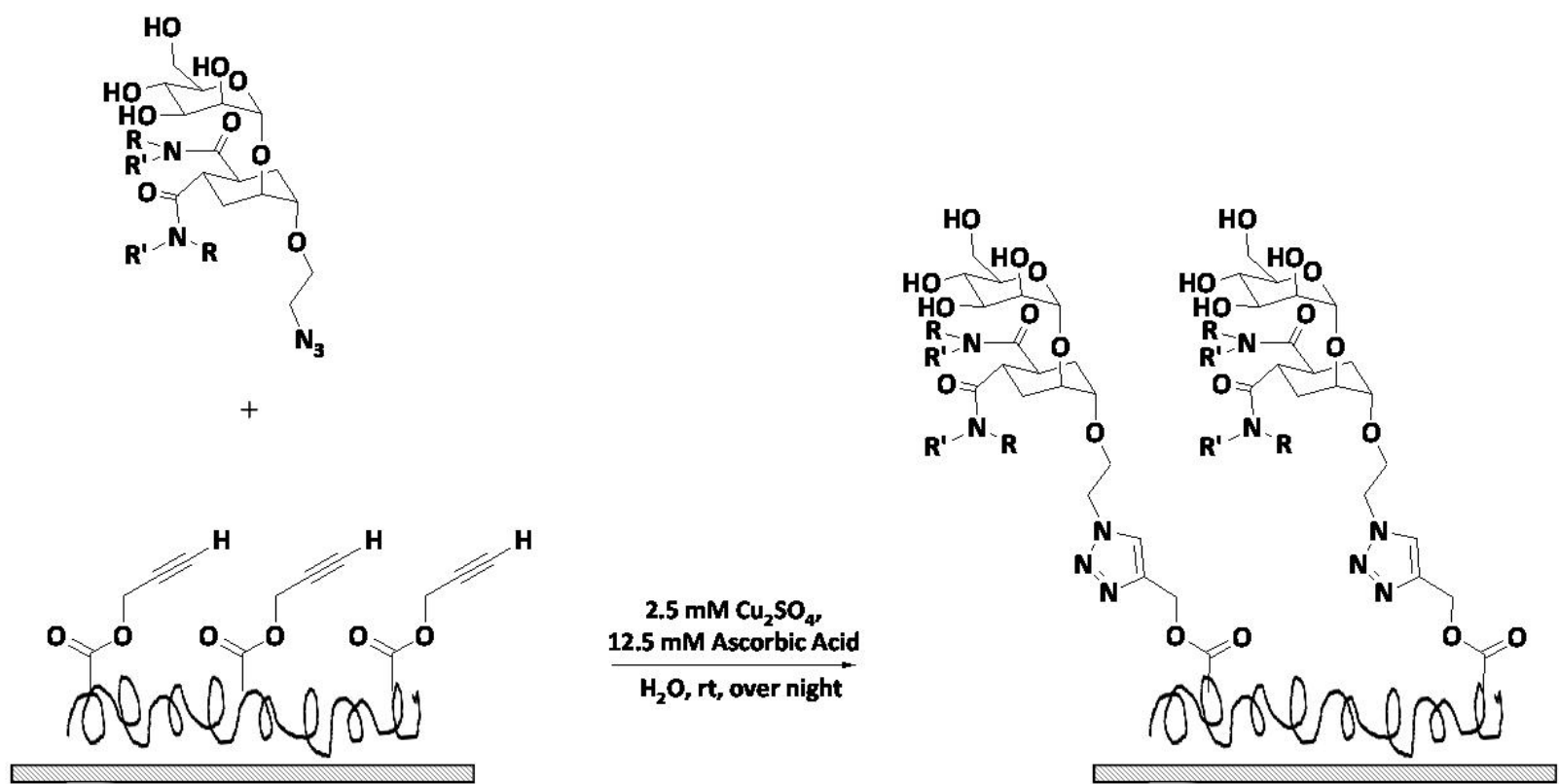

Figure 2 


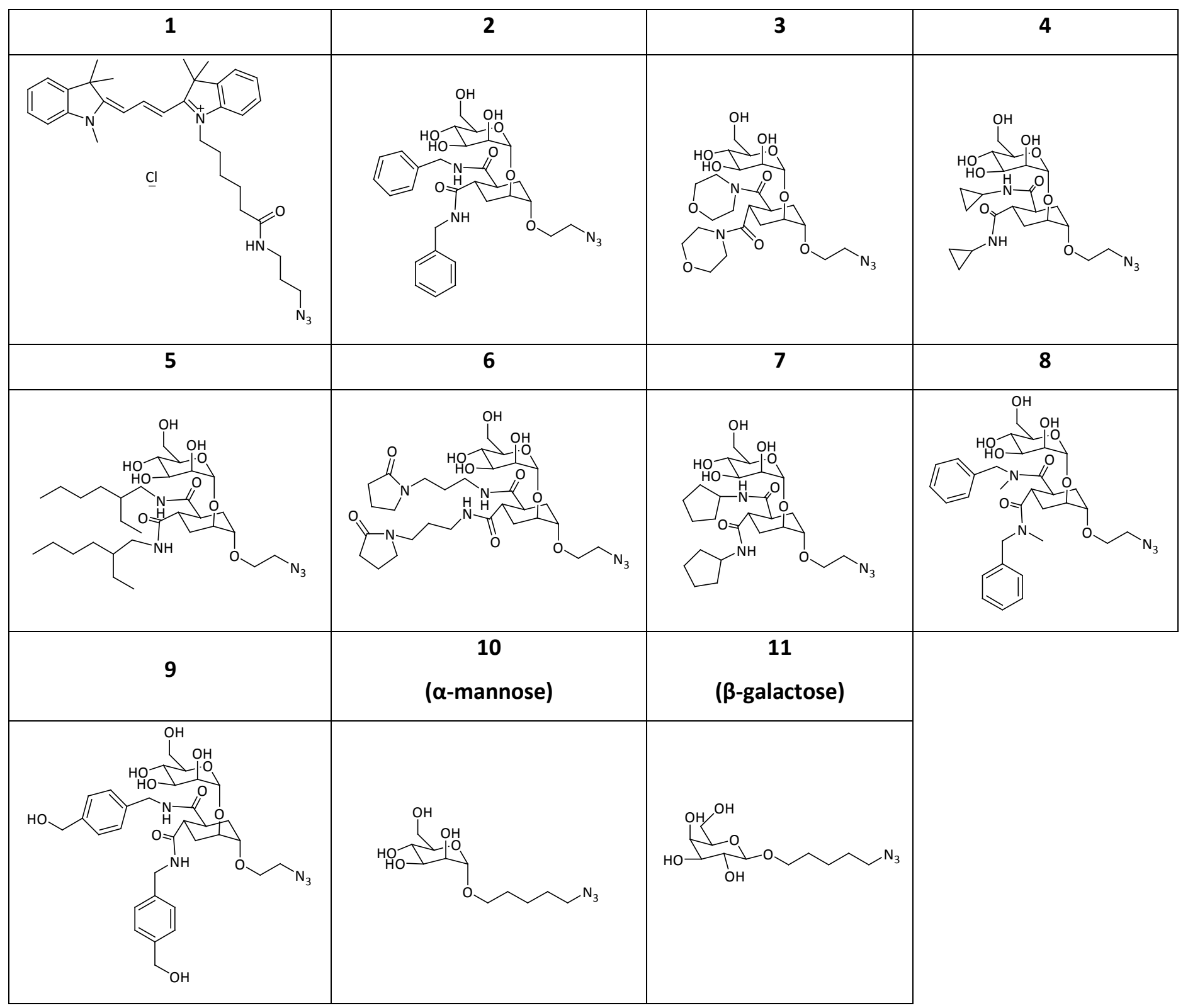

Figure 3 


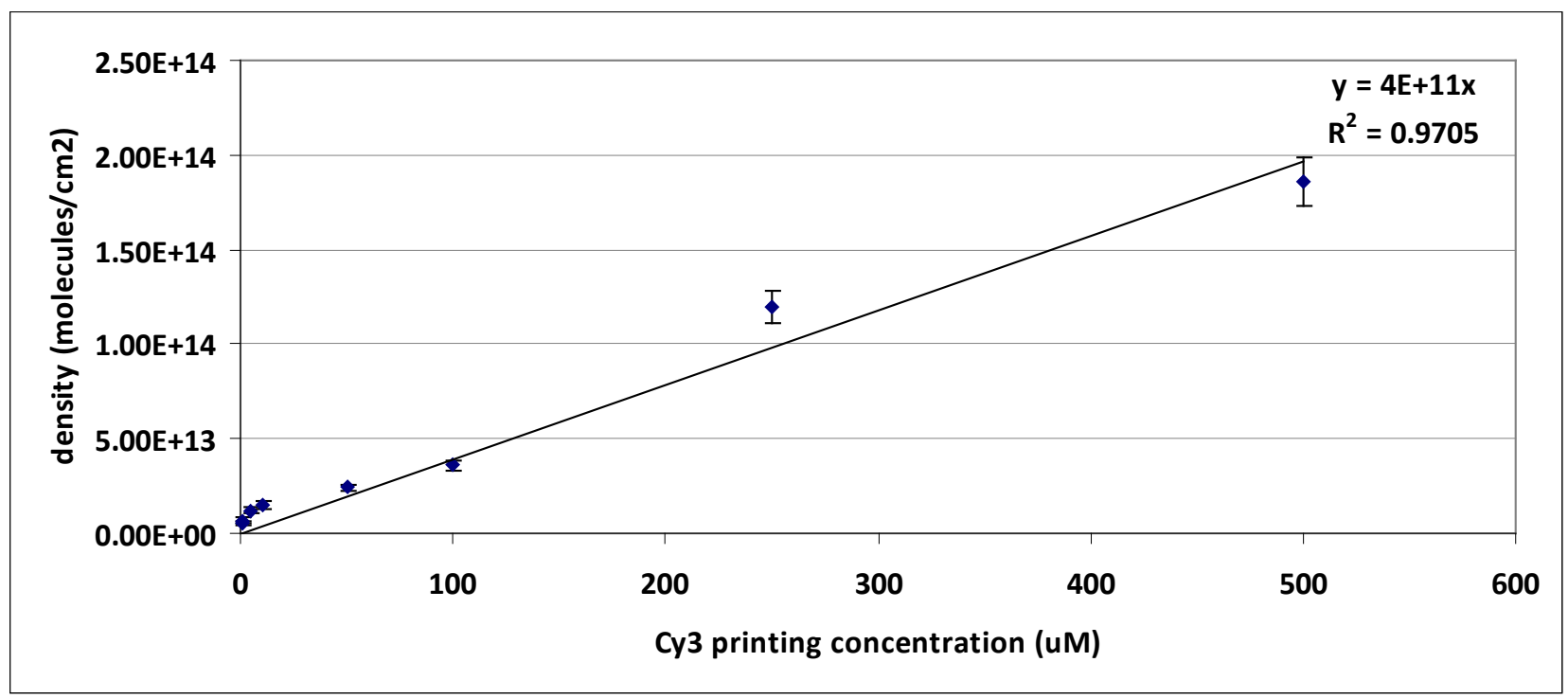

Figure 4 
(a)

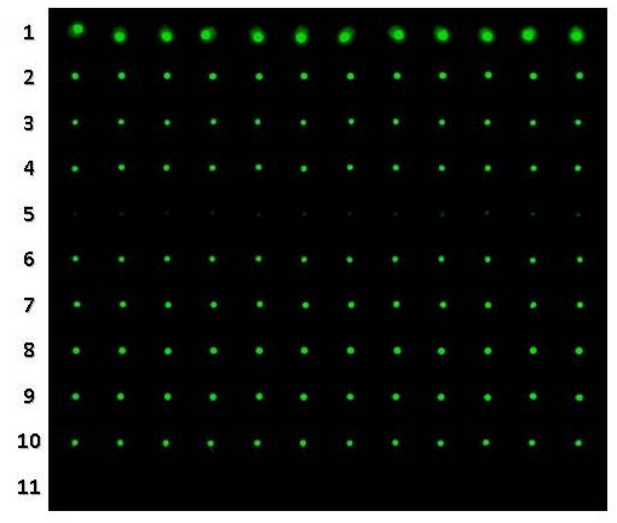

(b)

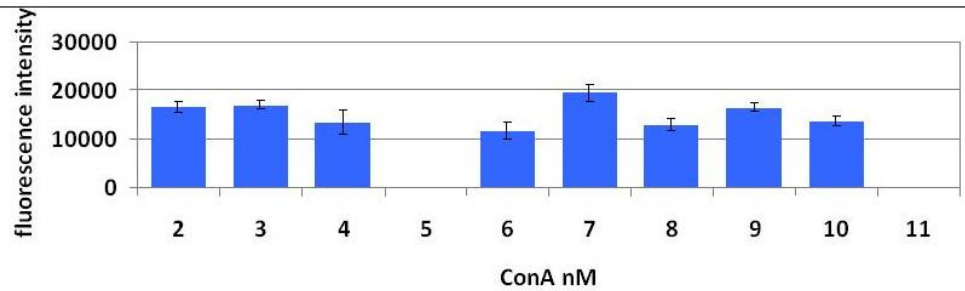

glycomimetics (50uM printing conc.)

Figure 5 

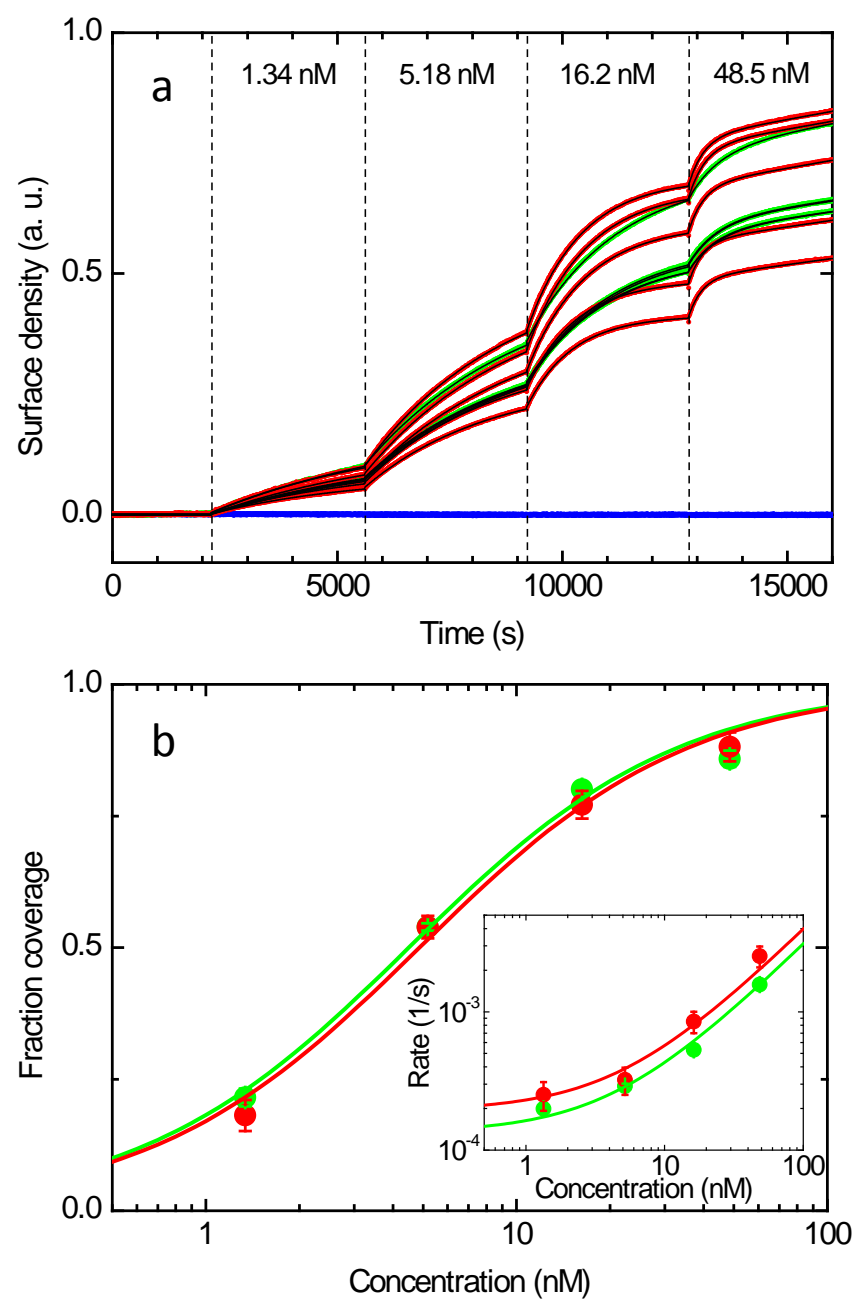

Figure 6 